\title{
ERWIN NYIREGYHÁZI
}

PSYCHOLOGISCHE ANALYSE EINES MUSIKALISCH HER= VORRAGENDEN KINDES

\section{VON}

DR. GÉZA RÉVÉSZ

PRIVATDOZENT AN DER UNIVERSITAT BUDAPEST

MIT NOTENBEILAGEN UND 4 FIGUREN IM TEXT

LEIPZIG ๑ VERLAG VON VEIT \& COMP. ๑ 1916 
Druck von Metzger \& Wittig in Lolpzig. 


\section{Meiner Magda}


